\title{
A PROPOSAL FOR THE DEVELOPMENT OF TECHNIQUES FOR THE IMPLEMENTATION OF CONTROL TO EXPERIMENTAL CHAOTIC SYSTEMS
}

Final Technical Report

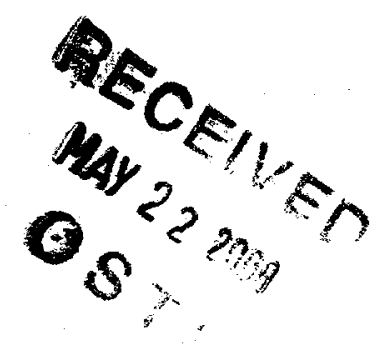

Celso Grebogi and Jaries Yorice

Principal Investigators

University of Maryland

College Park. MD 20742-3511

February 28. 2000

Prepared for the U.S. Department of Energy under Grant Number DE-FG02-93ER25166
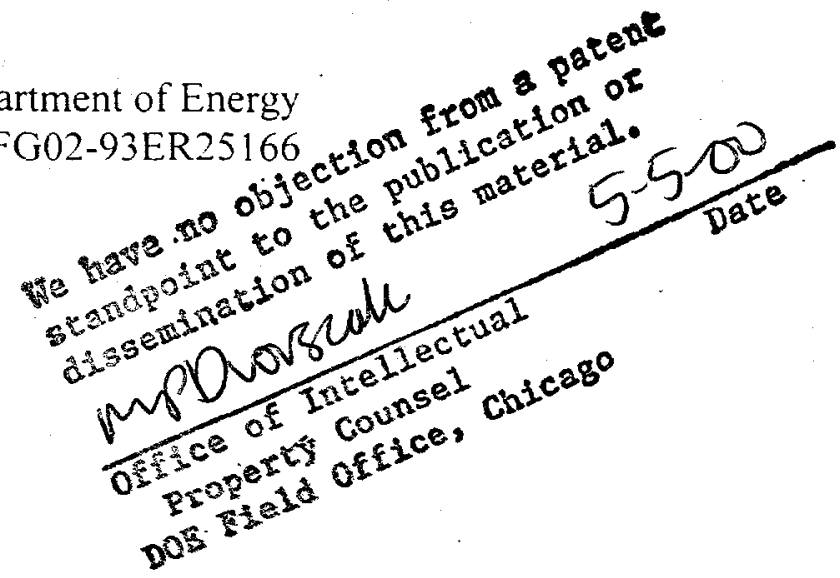


\section{DISCLAIMER}

This report was prepared as an account of work sponsored by an agency of the United States Government. Neither the United States Government nor any agency thereof, nor any of their employees, make any warranty, express or implied, or assumes any legal liability or responsibility for the accuracy, completeness, or usefulness of any information, apparatus, product, or process disclosed, or represents that its use would not infringe privately owned rights. Reference herein to any specific commercial product, process, or service by trade name, trademark, manufacturer, or otherwise does not necessarily constitute or imply its endorsement, recommendation, or favoring by the United States Government or any agency thereof. The views and opinions of authors expressed herein do not necessarily state or reflect those of the United States Government or any agency thereof. 


\section{DISCLAIMER}

Portions of this document may be illegible in electronic image products. Images are produced from the best available original document. 


\section{FINAL REPORT}

\section{CONTROLLING COMPLEXITY}

The following papers resulted from our proposed work in controlling complex dynamical systems. These systems include those with many attractors and complicated basin structures, to the point where relatively small perturbations can be used to move from one basin to another. Related to this area is our discovery of and subsequent work on Wada basin boundaries, where three or more attractors have basin boundaries that are intertwined so that every point on the boundary between two of the basins is also on the boundary of all other basins; such systems have unusual flexibility for the type of control considered here.

1. D. Auerbach and J. A. Yorke, Controlling chaotic fluctuations in semiconductor laser arrays, J. of the Optical Soc. of America B 13, 2178-2187 (1996).

2. U. Feudel, C. Grebogi, B. R. Hunt, J. A. Yorke, A map with more than 100 coexisting low-period periodic attractors, Phys. Rev. E 54, 71-81 (1996).

3. C. Grebogi and L. Poon, Controlling complex systems, Proc. Intern. Conf. on Nonlinearity, Bifurcation and Chaos (Lodz, Poland, 1996), pp. 22-27.

4. Y. C. Lai and C. Grebogi, Complexity in Hamiltonian-driven dissipative chaotic dynamical systems, Phys. Rev. E 54, 4667-4676 (1996).

5. H. E. Nusse and J. A. Yorke, Wada basin boundaries and basin cells, Physica D 90, 242-261 (1996).

6. H. E. Nusse and J. A. Yorke, Basins of attraction, Science 271, 1376-1380 (1996).

7. L. Poon, J. Campos, E. Ott, C. Grebogi, Wada basin boundaries in chaotic scattering, Int. J. Bifurc. Chaos 6, 251-266 (1996).

8. E. Barreto, B. R. Hunt, C. Grebogi, J. A. Yorke, From high dimensional chaos to stable periodic orbits: the structure of parameter space, Phys. Rev. Lett. 78 (1997), 4561-4564.

9. U. Feudel and C. Grebogi, Multistability and the control of complexity, Chaos 7 (1997), 597-604.

10. C. Grebogi and Y. C. Lai, Controlling chaos in high dimensions, IEEE Trans. Circ. Sys. 44 (1997), 971-975.

11. H. E. Nusse and J. A. Yorke, The structure of basins of attraction and their trapping regions, Ergod. Th. Dynam. Sys. 17 (1997), 463-482. 
12. Z. Toroczkai, G. Károlyi, A. Péntek, T. Tél, C. Grebogi, J. A. Yorke, Wada dye boundaries in open hydrodynamical flows, Physica A 239 (1997), 235-243.

13. L. Poon, U. Feudel, C. Grebogi, J. A. Yorke, Dynamical properties of a simple mechanical system with a large number of coexisting periodic attractors, Chaos, Solitons and Fractals 9 (1998), 171-180.

14. B. R. Hunt, J. A. C. Gallas, C. Grebogi, J. A. Yorke, H. Koçak, Bifurcation rigidity, Physica $\mathrm{D}$ (in press).

\section{COMMUNICATING WITH CHAOS}

The following papers concern our proposed work on using controlled chaotic signals for communication. For the transmitting site, we demonstrated how to use small perturbations of a dynamical system to achieve a desired coding sequence in its symbolic dynamics. For the receiving site, we discussed conditions under which a dynamical system with input from the transmitted signal will synchronize with the transmitter in order to output the desired symbol sequence. One important discovery we made was that by controlling the dynamics of the transmitter on an appropriate chaotic saddle, we can reduce the incidence of transmission errors without significantly degrading the throughput of the system, as measured by its topological entropy.

1. C. Grebogi, Y. C. Lai, S. Hayes, Chaos: control and communication, in Nonlinear Dynamics in Lasers, SPIE Proceedings, Vol. 2792, 1996, 15-29.

2. S. Hayes and C. Grebogi, Using controlled chaos for digital signaling: a method for one-stage complex waveform synthesis, Proc. of IEEE MTT-S Microwave Symposium, 1996.

3. K. G. Szabó, Y. C. Lai, T. Tél, C. Grebogi, Critical exponent for gap-filling at crisis, Phys. Rev. Lett. 77, 3102-3105 (1996).

4. E. Bollt, Y. C. Lai, C. Grebogi, Coding, channel capacity and noise resistance in communication with chaos, Phys. Rev. Lett. 79 (1997), 3787-3790. (1997).

5. C. Grebogi, Y. C. Lai, S. Hayes, Control and applications of chaos, Intern. J. Bif. and Chaos 7 (1997), 2175-2198 and J. Franklin Inst. 334B (1997), 11151146.

6. C. Grebogi, Y. C. Lai, S. Hayes, Controlling chaos with applications to nonlinear digital communication, in Proc. School of Biophysics (World Scientific, 1997).

7. B. R. Hunt. E. Ott, J. A. Yorke, Differentiable generalized synchronization of chaotic systems, Phys. Rev. E 55 (1997), 4029-4034.

8. E. Rosa, S. Hayes, C. Grebogi, Noise filtering in communication with chaos, Phys. Rev. Lett. 78 (1997), 1247-1250. 
9. M. S. Baptista, Cryptography with chaos, Phys. Lett. A 240 (1998), 50.

10. J. Jacobs, E. Ott, B. R. Hunt, Calculating topological entropy for transient chaos with an application to communicating with chaos, Phys. Rev. E 57 (1998), $6577-6588$.

11. E. Rosa, E. Ott, M. H. Hess, Transition to phase synchronization of chaos, Phys. Rev. Lett. 80 (1998), 1642-1645.

12. E. Rosa and E. Ott, Mixed basin boundary structures of chaotic systems, Phys. Rev. E 59 (in press).

\section{TARGETING}

In the following papers we have continued to explore the theory and applications of the method for controlling chaos we proposed in 1990, with particular emphasis on the problem of targeting a system from one state to another. Our original method of chaos control is to convert chaotic motion to one of the infinite number of unstable time-periodic motions contained within the chaotic attractor by making only small time-dependent perturbations to an available system parameter. In targeting, on the other hand, one rapidly takes the system state from an initial point to some specified targeting region in phase space by applying small controls. The purpose of targeting is not only to control the final state of a system, but also to drastically reduce the amount of time spent in a transient chaotic state before the controlled state is reached. Thus we have also considered the problems of optimizing cost (in time, energy, etc.) to reach a given final state, and of optimizing the controlled state. In the latter case, our main result is that the optimal controlled state is typically a low-period unstable periodic orbit, which validates the approach commonly taken for pragmatic reasons in applications of chaos control.

1. C. Grebogi and Y. C. Lai, Stabilizing unstable periodic orbits in chaotic dynamical systems using small feedback control, in Proceedings of the Institute of Electrical Engineers Waseda Workshop, Japan, 1996, 83-97.

2. B. R. Hunt and E. Ott, Optimal periodic orbits of chaotic systems, Phys. Rev. Lett. 76. 2254-2257 (1996).

3. B. R. Hunt and E. Ott, Optimal periodic orbits of chaotic systems occur at low period, Phys. Rev. E 54, 328-337 (1996).

4. P. So, E. Ott, S. J. Schiff, D. T. Kaplan, T. Sauer, C. Grebogi, Detecting unstable periodic orbits in chaotic experimental data, Phys. Rev. Lett. 76, 47054708 (1996).

5. E. Barreto, F. Casas, C. Grebogi, E. J. Kostelich, Control of chaos: impact oscillators and targeting, in Interaction Between Dynamics and Control in Advanced Mechanical Systems, ed. D. H. Van Campen (Kluwer, 1997), 17-26. 
6. E. Barreto, Y. C. Lai, C. Grebogi, Controlling chaos with applications to mechanical systems. in Nonlinear Dynamics of Material Processing and Manufacturing, ed. F. C. Moon (Wiley, New York, 1997), 285-302.

7. F. Casas and C. Grebogi, Control of chaotic impacts, Int. J. Bifurc. Chaos 7 (1997), 951-955.

8. C. Grebogi and Y. C. Lai, Controlling chaotic dynamical systems, Systems and Control Lett. 31 (1997), 307-312.

9. B. R. Hunt and E. Ott, Controlling chaos using embedded unstable periodic orbits: the problem of optimal periodic orbits, in Nonlinear Dynamics, Chaotic and Complex Systems, ed. Infeld, E., Zelazny, R., Galkowski, A. (Cambridge Univ. Press, 1997).

10. C. G. Schroer and E. Ott, Targeting in Hamiltonian systems that have mixed regular/chaotic phase spaces, Chaos 7 (1997), 512-519.

11. P. So, E. Ott, T. Sauer, B. J. Gluckman, C. Grebogi, S. J. Schiff, Extracting unstable periodic orbits from chaotic time series data, Phys. Rev. E 55 (1997), 5398-5417.

12. C. Grebogi and Y. C. Lai, Controlling chaos, chapter in the book Controlling Chaos, ed. H. G. Schuster (wiley, New York, 1998).

13. E. E. N. Macau, Targeting in chaotic scattering, Phys. Rev. E 57 (1998), 5337-5346 .

14. T. Tél, Y. C. Lai, C. Grebogi, Controlling transient chaos on chaotic saddles, chapter in the book Controlling Chaos, ed. H. G. Schuster (Wiley, New York, 1998).

15. F. T. Arecchi, S. Boccaletti, M. Ciofini, C. Grebogi, R. Meucci, The control of chaos: theoretical schemes and experimental realizations, Intern. J. Bif. Chaos (in press).

16. M. S. Baptista, Targeting applying e-bounded orbit correction perturbations, Int. J. Bifurc. Chaos (to appear).

\section{DETERMINISTIC MODELING}

The following papers resulted from our proposed study of deterministic modeling and its limitations. We have considered the extent to which the property of shadowing, known to hold for hyperbolic systems, holds also for nonhyperbolic systems, which are common in applications. Shadowing concerns how well a numerically computed trajectory of a dynamical system compares with an exact trajectory of the system, and also how differences between a physical system and a deterministic model can make 
the evolution of the system differ from that of the model. We have identified specific mechanisms that can cause shadowing to fail, and offered methods for quantifying the time scale over which shadowing can still be expected to hold in the presence of nonhyperbolicity. We have also considered other phenomena that are impediments to predictability in deterministic modeling. In particular, we have continued to investigate the incidence and properties of riddled and intermingled basins, in which a dynamical system with a symmetry can have multiple attractors whose basins of attraction are intertwined at all scales; if there is any uncertainty in the initial condition of a trajectory, no matter how small, then each of the attractors has a positive probability of attracting the trajectory. In addition, we have also addressed basic questions of model building using experimental data, and, in particular, the theory of embedding for chaotic dynamical systems.

1. J. C. Alexander, B. R. Hunt, I. Kan, J. A. Yorke, Intermingled basins for the triangle map, Ergod. Th. Dynam. Sys. 16, 651-662 (1996).

2. E. Kostelich, J. A. Yorke, Z. You, Plotting stable manifolds: error estimates and noninvertible maps, Physica D 93, 210-220 (1996).

3. Y. C. Lai and C. Grebogi, Characterizing riddled fractal sets, Phys. Rev. E 53, 1371-1374 (1996).

4. Y. C. Lai, C. Grebogi, J. A. Yorke, S. C. Venkataramani, Řididing bifurcation in chaotic dynamical systems, Phys. Rev. Lett. 77, 55-58 (1996).

5. Y. C. Lai and C. Grebogi, Noise-induced riddling in chaotic dynamical systems, Phys. Rev. Lett. 77, 5047-5050 (1996).

6. A. Lasota and J. A. Yorke, When the long-time behavior is independent of the initial density. SIAM J. Math. Anal. 27, 221-240 (1996).

7. J. C. Sommerer and E. Ott, Intermingled basins of attraction: uncomputability in a simple physical system, Phys. Lett. A 214, 243 (1996).

8. S. C. Venkataramani, T. M. Antonsen, E. Ott, J. C. Sommerer, On-off intermittency: power spectrum and fractal properties of time series, Physica D 96. 66 (1996).

9. S. C. Venkataramani. B. R. Hunt, E. Ott, Bubbling transition, Phys. Rev. E 54. 1346-1360 (1996).

10. S. C. Venkataramani, B. R. Hunt, E. Ott, D. J. Gauthier, J. C. Bienfang, Transitions to bubbling of chaotic systems, Phys. Rev. Lett. 77, 5361-5364, (1996).

11. B. R. Hunt and V. Yu. Kaloshin, How projections affect the dimension spectrum of fractal measures, Nonlinearity 10 (1997), 1031-1046. 
12. J. Jacobs, E. Ott, C. Grebogi, Computing the measure of non-attracting chaotic sets, Physica D 108 (1997), 1-11.

13. E. J. Kostelich, I. Kan, C. Grebogi, E. Ott, J. A. Yorke, Unstable dimension variability: a source of nonhyperbolicity in chaotic systems, Physica D 109 (1997), 81-90.

14. Y. C. Lai, C. Grebogi, J. A. Yorke, Intermingled basins and riddling bifurcation in chaotic dynamical systems, in US-Chinese Conference on Recent Developments in Differential Equations and Applications (1997), 138-163.

15. T. Sauer, C. Grebogi, J. A. Yorke, How long do numerical chaotic solutions remain valid? Phys. Rev. Lett. 79 (1997), 59-62.

16. T. Sauer and J. A. Yorke, Are the dimensions of a set and its image equal under typical smooth functions?, Ergod. Th. Dynam. Sys. 17 (1997), 941-956

17. L. Poon, C. Grebogi, T. Sauer, J. A. Yorke, Shadowing in chaotic dynamical systems, in Handbook of Dynamical Systems, ed. B. Fiedler (1998).

18. T. D. Sauer, J. A. Tempkin, J. A. Yorke, Spurious Lyapunov exponents in attractor reconstruction, Phys. Rev. Lett. 81 (1998), 4341-4344.

19. C. G. Schroer, E. Ott, J. A. Yorke, Effect of noise on nonhyperbolic chautic attractors, Phys. Rev. Lett. 81 (1998), 1397-1400.

20. C. G. Schroer, T. Sauer, E. Ott, J. A. Yorke, Predicting chaos most of the time from embeddings with self-intersections, Phys. Rev. Lett. 80 (1998), 1410-1413.

21. Y. C. Lai and C. Grebogi, Riddling in classical mechanical systems, Appl. Mech. Rev. (in press).

22. Y. C. Lai, C. Grebogi, J. Kurths, Modeling of deterministic chaotic systems, Phys. Rev. E 59 (in press).

\section{OTHER RESEARCH}

Other research supported by DOE over the last three years, reflected by the following papers, includes among other topics work on: the interface between chaotic and stable periodic behavior as system parameters vary; bifurcations of non-smooth systems that describe impact oscillators: phenomena that occur in quasiperiodically forced systems; and the fractal and topological properties of chaotic invariant sets, in particular those arising in fluid flow.

1. F. Casas, W. Chin, C. Grebogi, E. Ott, Universal grazing bifurcations in impact oscillators. Phys. Rev. E 53, 134-139 (1996). 
2. B. R. Hunt, Estimating invariant measures and Lyapunov exponents, Ergod. Th. Dynam. Sys. 16, 735-749 (1996).

3. B. R. Hunt, K. M. Khanin, Ya. G. Sinai, J. A. Yorke, Fractal properties of critical invariant curves, J. Stat. Phys. 85, 261-276 (1996).

4. B. R. Hunt, E. Ott, J. A. Yorke, Fractal dimensions of chaotic saddles of dynamical systems, Phys. Rev. E 54, 4819-4823 (1996).

5. B. R. Hunt, Maximum local Lyapunov dimension bounds the box dimension of chaotic attractors, Nonlinearity 9, 845-852 (1996).

6. Y. C. Lai, U. Feudel, C. Grebogi, Scaling behaviors in the transition to chaos in quasiperiodically driven dynamical systems, Phys. Rev. E 54, 6070-6073 (1996).

7. B. Peratt and J. A. Yorke, Continuous avalanche mixing of granular solids in a rotating drum, Europhys. Lett. 35, 31-35 (1996).

8. S. Banerjee, C. Grebogi, E. Ott, J. A. Yorke, Border collision bifurcations in power electronics circuits, Proc. Int. Conf. on Dynamical Systems, Bangalore, India (1997).

9. S. Banerjee, C. Grebogi, E. Ott, J. A. Yorke, Anomalous bifurcations in dcde converters: borderline colitisions in piecewise smooth maps, Proc. Power Electronics Specialists Conf., St. Louis, Missouri, 1997.

10. W. Chin, B. R. Hunt, J. A. Yorke, Correlation dimension for iterated function systems, Trans. Amer. Math. Soc. 349 (1997), 1783-1796.

11. M. Ding, C. Grebogi, J. A. Yorke, Chaotic dynamics, in The Impact of Chaos on Science and Society, ed. C. Grebogi and J. A. Yorke (United Nations University Press, Tokyo, 1997), 1-17.

12. U. Feudel, C. Grebogi, E. Ott, Phase-locking in quasiperiodically forced systems, Phys. Reports 290 (1997), 11-25.

13. B. R. Hunt and E. Ott, Structure in the parameter dependence of order and chaos for the quadratic map, J. Phys. A 30 (1997), 7067-7076.

14. J. Jacobs. E. Ott, T. Antonsen, J. A. Yorke, Modeling fractal entrainment sets of tracers advected by chaotic temporally irregular fluid flows using random maps, Physica D 109 (1997), 1723.

15. J. Jacobs, B. R. Hunt and E. Ott, Scaling of the durations of chaotic transients in windows of attracting periodicity, Phys. Rev. E 56 (1997), 6508-6515.

16. J. Kennedy and J. A. Yorke, The topology of stirred fluids, Topology \& Appl. 80 (1997), 201-238. 
17. Y. C. Lai, Y. Nagai, C. Grebogi, Characterization of the natural measure by unstable periodic orbits in chaotic attractors, Phys. Rev. Lett. 79 (1997), 649652.

18. M. A. F. Sanjuán, J. nennedy, C. Grebogi, J. A. Yorke, Indecomposable continua in dynamical systems with noise: fluid flow past an array of cylinders, Chaos 7 (1997), 125-138.

19. M. A. F. Sanjuán, J. Kennedy, E. Ott, J. A. Yorke, Indecomposable continua and the characterization of strange sets in nonlinear dynamics, Phys. Rev. Lett. 78 (1997), 1892-1895.

20. K. T. Alligood and J. A. Yorke, Rotation intervals for chaotic sets. Proc. Amer. Math. Soc. 126 (1998), 2805-2810.

21. S. Banerjee, C. Grebogi, J. A. Yorke, Robust chaos, Phys. Rev. Lett. 80 (1998), 3049-3052.

22. B. R. Hunt, The Hausdorff dimension of graphs of Weierstrass functions, Proc. Amer. Math. Soc. 126 (1998), 791-800.

23. U. Feudel, A. Witt, Y. C. Lai, C. Grebogi, Basin bifurcation in quasiperiodically forced systems, Phys. Rev. E 58, 3060-3066 (1998).

24. B. Peratt and J. A. Yorke, Modeling continuous mixing of granular solids in a rotating drum, Physica D 118 (1998), 293.

25. C. Robert, K. T. Alligood, E. Ott, J. A. Yorke, Outer tangency bifurcations of chaotic sets, Phys. Rev. Lett. 80 (1998), 4867-4870.

26. Z. Toroczkai, G. Károlyi, A. Péntek, T. Tél, C. Grebogi, Advection of active particles in open chaotic flows, Phys. Rev. Lett. 80 (1998), 500-503.

27. G. H. Yuan, S. Banerjee. J. A. Yorke, Border-collision bifurcations in the Buck converter, IEEE Trans. Circ. Sys. 45 (1998), 707.

28. S. Banerjee, C. Grebogi, E. Ott, Border collision bifurcations: an explanation of the observed bifurcation phenomena in the boost converter, IEEE Trans. Power Electronics (in press).

29. A. Witt, R. Braun. F. Feudel. C. Grebogi, J. Kurths, Tracer dynamics in a flow of driven vortices, Phys. Rev. E 59 (in press).

\subsubsection{BOOKS}

The following books resulted in part from research supported by DOE over the last 16 years.

1. K. T. Alligood, T. Sauer. J. A. Yorke, Chaos: an Introduction to Dynamical Systems, Springer-Verlag. 1996. 
2. C. Grebogi and J. A. Yorke, eds., The Impact of Chaos on Science and Society, United Nations University Press, Tokyo, 1997.

3. H. E. Nusse and J. A. Yorke, Dynamics: Numerical Explorations, Second, Revised and Expanded Edition, 608 + xvi pp., accompanied by software program Dynamics (Version 2), an interactive program for IBM compatible PCs and Unix computers, by H. E. N. and J. A. Y. (the Unix version of the program is by B. R. Hunt and E. J. Kostelich), Springer-Verlag, New York, 1998.

4. E. Ott, Chaos in Dynamical Systems, Cambridge University Press, 1993.

5. E. Ott, T. Sauer, J. A. Yorke, Coping with Chaos, Wiley, New York, 1994. 